\title{
A Study on Routing Protocols in Wireless Sensor Network
}

\author{
Deepa $\mathrm{H}$ \\ Calcutta Institute of Engineering and Management \\ 24/1A Chandi Ghosh Road, Kolkata-700040
}

\author{
Ayan Kumar Das \\ Calcutta Institute of Engineering and Management \\ 24/1A Chandi Ghosh Road, Kolkata-700040
}

\begin{abstract}
Wireless Sensor Network (WSN) consists of many small sensor nodes. A sensor node is a battery operated small device capable of computation and communication. These nodes are capable of sensing events within their coverage area. The main objective of WSN is to sense destructive events from its environment and send that information to the sink node, so that it can take the corrective actions. As the sensor nodes are deployed in remote location, it is very difficult to recharge the batteries. Thus energy efficiency is the main challenge in WSN. The paper gives a brief study about different energy efficient routing protocols, so that the problem areas can be identified and solved.
\end{abstract}

\section{General Terms}

Energy Efficient Routing Protocols

\section{Keywords}

Wireless sensor network, routing, network lifetime, power management.

\section{INTRODUCTION}

The deployment of Wireless Sensor Network in various applications has paved way for advancement in many fields in the recent years. Different techniques for routing and power management have been designed for the efficient use of the new technology. The basic idea behind the development of most Wireless Sensor Networks is that it must remain operational for longer lifetime on limited energy resources. The constraints relating to the sensor nodes is a great issue basically due to their limited energy, storage capacity and computation power. These constraints can be taken into account to route the sensed data efficiently from source node to sink node. There are a number of existing routing protocols which can meet the requirement. The paper involves discussions related to various such algorithms based on power aware routing, agent based routing, query driven routing and clustering algorithms. The comparative study would allow one to determine the compatibility of the given algorithms pertaining to their characteristics. These protocols can be further categorized according to various key factors in a systematic manner which would help in the proper assessment of their respective parameters, ultimately leading to a better power management scheme and ensuring maximum network lifetime. In the next part a review work of existing algorithms is given, followed by a comparative study and conclusion part.

\section{REVIEW WORK}

In WSN, to send the information about devastating events from source node to the base station in an energy efficient way, many algorithms have been designed. The packets can be sent by shortest path. Though time can be saved, the problem of nodes in the path going down very early, still persists. To solve the problem, multipath routing techniques are designed. As sensor nodes are deployed very densely, here all the nodes of a particular region will try to send the same data by different paths. Thus actually sending duplicate data causes wastage of energy and network congestion. Clustering algorithm can be a better solution for this and also increases the lifetime of the network. Here we have discussed a number of energy efficient algorithms. The comparison and assessment of various parameters used in different algorithms would give an idea as to which one suits best under which category thereby guiding in the optimum allocation of given resources.

\subsection{Cluster Based Algorithm}

\subsubsection{EEHC (Energy Efficient Hierarchical Clustering)}

This algorithm defines a new criterion [1,2,3] for non- cluster nodes to choose the Cluster Head $(\mathrm{CH})$ depending on residual energy and size of clusters. Here, initially data aggregation takes place where sensor nodes are aggregated into smaller clusters. Non cluster node examines the residual energy and size of clusters to form a group of clusters, based on distance between the nodes. The nodes in clusters calculate their own degree and broadcast it in the network. Now the source node broadcasts a message containing the requisite amount of energy. So nodes in each level compare it with their own energy level and the efficient one is elected as $\mathrm{CH}$. In this way efficient clusters are ordered according to decreasing order of energy level. Now $\mathrm{CH}$ will broadcast to the neighbors about its election and build multi-hop paths to source node. $\mathrm{CH}$ will choose the farthest node that has most energy in next hop. Here, it based on the fact that with the decrease in size of clusters, the distance between respective nodes decreases as well. As a result, when the distance between the clusters increase, the energy spent increases to a great extent. This drawback can be ignored when other factors are taken into consideration. In the presence of heterogeneous nodes, this protocol ensures increase in the lifetime and robustness of the network.

\subsubsection{HEED (Hybrid Energy Efficient Distribution)}

As the name suggests, HEED [4,5] is: Hybrid- clustering is based on two parameters that is, residual energy and communication cost; Energy Efficient- elects $\mathrm{CH}$ that are rich in residual energy and; Distributed- re-clustering results in distributing energy consumption. Here, initially each node sends an election message including node ID or cost to each of its neighbors, and receives the same from its neighbors. Each node checks to see if there are some $\mathrm{CHs}$ around. In this process, one node in each cluster acts as a $\mathrm{CH}$ so as to coordinate other nodes. This ensures the production of compact clusters which are properly distributed, hence 
stabilizing individual as well as neighboring nodes. This protocol does not need to be aware of the location of nodes and undergoes simple local communication. Thus HEED is fast and has low overhead. It is independent of homogeneous nodes. Energy is distributed efficiently, that ensures maximum lifetime of the network. The only disadvantage is that HEED depends on network topology.

\subsubsection{MRECA (Mobility Resistant Efficient Clustering Approach)}

This algorithm functions in a similar manner as that of HEED, with minimal complexities. Here, it is assumed that the locations of nodes are unaware. The cluster head broadcasts local information and the respective score value in the network. The score is used to calculate the delay for cluster head announcement. This algorithm [6] is based on the mobility resistant technique involving deterministic time without iterations. Such an approach ensures better energy efficiency. The main feature is increased speed of clustering and robustness of the network. Though MRECA determines when the routing decision is made by the nodes, it does not focus on how the same is performed. Another drawback involved here is that the inter-cluster communication is not considered.

\subsubsection{LEACH (Low Energy Adaptive Clustering Hierarchy)}

LEACH [7] is a homogeneous clustering algorithm. It distributes the consumed energy throughout the entire network, where the network is divided into clusters and equivalent cluster heads in proper manner. The run time of network is divided into many rounds efficiently and it lets the data transmission phase last for a fixed period of time, thus nodes in the network consume energy in a balanced manner thereby prolonging the lifetime of the network. This method treats all nodes without discrimination, hence the stability period of LEACH is very short and the nodes die at a steady rate. LEACH forms only single-hop clusters, which could increase the complexity of routing. As such, its performance is not suited for heterogeneous wireless sensor networks as it fails to take full advantage of the extra energy provided by the heterogeneous nodes.

\subsubsection{DEEC (Distributed Energy Efficient Clustering)}

This protocol is basically used in heterogeneous wireless sensor networks. The main focus here $[8,9]$ is on saving more energy for the heterogeneous networks such that each node transmits sensed data to the base station through cluster head. The cluster head election along with the further processing is done in an energy efficient manner. The nodes with high initial and residual energy will be preferred over the nodes with low energy during the process of cluster head election. So as to control the energy expenditure of each node, DEEC uses average energy of the network as the reference energy. Thus DEEC can also prolong the network lifetime and inhibit more effective messages. When compared to LEACH, it could be seen that DEEC is more efficient.

\subsection{Power Aware Routing Algorithm}

\subsubsection{MECN (Minimum Energy Communication Network)}

MECN is a location based protocol which aims at acquiring minimum amount of energy for the randomly deployed network. The routing process $[10,11]$ involves two phases- the construction of enclosure graph and; the cost distribution process. It computes the optimal path to be traversed and takes in minimum power paths from each sensor. Thus, in this way it creates a spanning tree by which energy dissipated in traversing the network is reduced. This can be called as the minimum power topology. The cost distribution is also done in an efficient manner where the cost of each node is considered as the minimum amount of power required by the nodes to transfer sensed data, so as to create a direct path to the base station. A notable feature of MECN protocol is that it is self reconfiguring, that is it can dynamically adjust itself to node failure if any.

\subsubsection{PEGASIS (Power Efficient Gathering in Sensor Information System)}

PEGASIS [12] is yet another routing protocol which can be considered more efficient than previously described LEACH protocol. It outdoes LEACH's performance by removing the overhead of dynamic cluster formation and using only one transmission to the base station per round. In PEGASIS instead of forming multiple clusters, a chain of nodes is constructed which ensures local coordination among neighboring sensor nodes and hence reduces the bandwidth of communication. This helps in increasing the lifetime of each sensor nodes which results in an overall efficiency with respect to network lifetime. The notable drawback that persists in PEGASIS is that all sensor nodes are required to maintain a database with the location of all nodes in the entire network. Such a requirement results in an increased overhead.

\subsubsection{MTE (Minimum Transmission Energy)}

The MTE protocol [13] is basically used for the transmission of data to long distance. The sensor nodes route the sensed data to the base station through intermediate nodes. These intermediate nodes are chosen carefully based on power management policy in a way by which the energy required to transmit the data is minimized. Thus in this protocol multi hop communication takes place where the intermediate nodes act as the basic router. At every node, reduction of transmission power is ensured. The nodes that are closest to the sink would be used more regularly which causes the early death for those nodes. The main drawback here is that, large amount of energy is dissipated if the nodes are close to each other.

\subsubsection{GEAR (Geographic and Energy Aware Routing)}

As the name suggests, this protocol $[14,15]$ uses geographic information so as to route the requested data. Here, the procedure of selecting neighbor nodes is done in an energy efficient manner so as to forward data towards the destination node. This process of forwarding data to the destination involves two phases. Firstly, as soon as it detects a neighbor, all the routes from the source node to the destination are determined and they are updated in the routing table. Secondly, it disperses the data packets within the region. Now the data is broadcasted to the previously detected neighbors in a way such that each node is made to know its own location as well as the neighbor's location. The process continues till these packets reach the destination. Thus instead of sending data to the entire network, the main idea here is to send the sensed data only to specific regions. With this remarkable feature, the protocol helps to save energy and power. 


\subsection{Agent Based Routing Algorithms}

\subsubsection{ABMR (Agent Based Multipath Routing)}

This algorithm works on the basis of parameters such as energy, multi-hop paths and reliability. Here, along with the traditional routing process, external agents are used to route data packets from the source node to the destination. This helps in the reduction of overhead of messages that are to be sent out. It involves building of reliable multiple paths from source to destination, in a way by which agents are usually transferred from one node to another so as to aggregate the sensed data. Agent Based Multipath Routing [16] involves the use of agents which collect the required information that include distance between the sensors as well as the residual energy. After this, the agent returns to the sender to decide the best number of nodes to be selected from amongst the neighbors. In short, the agents are transferred to the data source and the collected data is aggregated instead of moving the data to a centralized node for processing. Agents are used for identifying the faulty nodes for further processing. They help to reduce communication cost over very low bandwidth links among the sensors. Hence it simplifies the complexity of the routing algorithm.

\subsubsection{EEABR (Energy Efficient Ant Based Routing)}

The main objective of this algorithm is to assist in the prolongation of the network lifetime along with the improvement in quality of service. EEABR $[17,18]$ uses a colony of artificial ants which searches for prescribed path between the source node and destination node in a way such that the path is short in length as well as energy efficient. After traversing through a path, when these ants reach the destination node, they travel back through the path previously constructed and update the same based on the remaining energy and the number of nodes on the path. In this way, it builds a routing tree with optimized energy nodes. Thus we can say that EEABR is a self organizing and robust protocol where there is no need for defining path recovery algorithms. Also this algorithm is optimized in terms of distance and energy utilization, which ensures maximization of the network lifetime and minimization of communication load.

\subsubsection{ADHOP (Ant-based Dynamic Hop)}

It is an agent based reactive routing protocol where routes are set up on demand, when data is to be routed to an unknown destination. The protocol is self-configuring and uses dynamic hops so as to make efficient routing decision in the wireless network. Here all routing decisions are made in a way such that the nodes are not required to know the entire route for data transmission. This decision is made by the intermediary ants which guide the nodes to take decision about the best route that could be taken towards the destination. Thus the nodes are just required to direct the data to the next hop as per the local information. The dynamic nature of the protocol allows itself to easily adapt to any network requirements and topology changes. The nodes become attentive when any topology change is seen. Also it helps in the reduction of network overhead significantly.

\subsubsection{JARA (Jumping Ant Routing Algorithm)}

This agent based protocol $[20,21]$ is based on the combination of both proactive as well as reactive routing scheme. Firstly, this algorithm describes the use of proactive routing protocol by each node so as to maintain the topology of the predetermined hops, where the destination of a known path can be reached. Secondly, it focuses on reactive routing scheme in which the respective nodes use the ants so as to discover unknown destination outside the prescribed path. Each node updates itself with detailed information regarding neighbor nodes with the predetermined hops. JARA too is self configuring, and using this protocol, the path discovery can be done easily thus speeding up the entire process.

\subsection{Query Based Routing Algorithm- Rumor Routing}

This routing protocol $[22,23]$ helps to route data towards a destination especially when the geographical location is unavailable. It finds the shortest path between the query generating node and source nodes. Query data are flooded throughout the network. At first, the queries are scattered randomly throughout the entire network. Then these queries are routed along the paths generated by the agents. Here, each node in the network is required to maintain the list of its neighbors and a table of events such that the information can be broadcasted throughout the network. With respect to the obtained information the agents create the requisite path by ensuring that the overhead involved in the process is minimized. With the decrease in the number of queries flooded, the energy consumed decreases too. This protocol ensures robustness of the network as it helps to handle node failure, thus assuring maximization of network lifetime. 
Table1: Comparison of Different Routing Protocols

\begin{tabular}{|c|c|c|c|c|c|c|c|c|c|}
\hline Protocols & Mobility & $\begin{array}{c}\text { Power } \\
\text { Management }\end{array}$ & $\begin{array}{l}\text { Network } \\
\text { Lifetime }\end{array}$ & Scalability & $\begin{array}{c}\text { Resource } \\
\text { Awareness }\end{array}$ & Classification & $\begin{array}{l}\text { Data } \\
\text { Aggre- } \\
\text { gation }\end{array}$ & $\begin{array}{l}\text { Query } \\
\text { Based }\end{array}$ & $\begin{array}{l}\text { Multi } \\
\text { Path }\end{array}$ \\
\hline EEHC & Fixed & Maximum & $\begin{array}{l}\text { Very } \\
\text { Good }\end{array}$ & Very Good & Yes & Clustering & Yes & Yes & Yes \\
\hline HEED & Fixed & Maximum & $\begin{array}{l}\text { Very } \\
\text { Good }\end{array}$ & Limited & Yes & Hybrid & Yes & Yes & No \\
\hline MRECA & Limited & Maximum & $\begin{array}{l}\text { Very } \\
\text { Good }\end{array}$ & Very Good & Yes & Clustering & No & Yes & Yes \\
\hline LEACH & Fixed & Maximum & $\begin{array}{l}\text { Very } \\
\text { Good }\end{array}$ & Good & Yes & $\begin{array}{l}\text { Homogeneous } \\
\text { Clustering }\end{array}$ & No & No & No \\
\hline DEEC & Fixed & Maximum & $\begin{array}{l}\text { Very } \\
\text { Good }\end{array}$ & Very Good & Yes & $\begin{array}{l}\text { Heterogeneous } \\
\text { Clustering }\end{array}$ & No & Yes & Yes \\
\hline MECN & Supported & Maximum & Good & Very Good & Yes & Power Aware & No & No & No \\
\hline PEGASIS & Fixed & Maximum & $\begin{array}{l}\text { Very } \\
\text { Good }\end{array}$ & Limited & Yes & Power Aware & Yes & Yes & No \\
\hline MTE & Supported & Maximum & Good & Limited & Yes & Power Aware & No & No & Yes \\
\hline GEAR & Supported & Maximum & $\begin{array}{l}\text { Very } \\
\text { Good }\end{array}$ & Very Good & Yes & Power Aware & Yes & Yes & Yes \\
\hline ABMR & Supported & Maximum & Good & Good & Yes & Agent Based & Yes & Yes & Yes \\
\hline EEABR & Supported & Maximum & $\begin{array}{l}\text { Very } \\
\text { Good }\end{array}$ & Good & Yes & Query Based & No & Yes & Yes \\
\hline ADHOP & Supported & Limited & Good & Good & Yes & Agent Based & No & Yes & Yes \\
\hline JARA & Supported & Limited & Good & Good & Yes & Agent Based & No & Yes & Yes \\
\hline RUMOR & Fixed & Maximum & Good & Very Good & Yes & Query Based & Yes & Yes & Yes \\
\hline
\end{tabular}

\section{CONCLUSION}

Energy efficiency is the main research area in wireless sensor network. As sensor nodes have limited power sources, saving and recharging of power in batteries is a very important aspect. Energy saving provides a potential solution to this problem. Though developments in recent technologies have helped to decrease the power consumption, the lifespan of a wireless sensor network depends on the type of events that are to be dealt with. Other major limitations include decreased computation speed, high cost and limited bandwidth that must be shared among all nodes in the network. Different energy efficient techniques can be used according to different environmental conditions and requisite parameters. In this paper, a comparison of various such techniques has been presented, which would help one to know about the existing routing protocols and choose the optimum resources to deal with them. These protocols can be put under the respective category depending on their advantages and limitations, and utilized efficiently. Thus, the comparative study would be helpful in identifying the key characteristics of the protocols and the problems pertaining to them, so as to extend the research work and come up with more energy efficient routing techniques to improve the functionality of wireless sensor networks.

\section{REFERRENCE}

[1] Dilip Kumar, Trilok C.Aseri, R.B. Patel, "EEHC: Energy efficient heterogeneous clustered scheme for wireless sensor networks", Computer Communications 32.4: 662$667,2009$.
[2] Shio Kumar Singh, M P Singh, D K Singh, "A Survey of Energy-Efficient Hierarchical Cluster-Based Routing in Wireless Sensor Networks", Int. J. of Advanced Networking and Applications, Volume: 02, Issue: 02, Pages: 570-580, 2010.

[3] Rathna and Sivasubramanian, "Improving Energy Efficiency in Wireless Sensor Networks through Scheduling and Routing”, Research Scholar, Sathyabama University, TamilNadu, India, International Journal of Advanced Smart Sensor Network Systems, vol. 2, no.1, pp. 21-27, January 2012.

[4] Hesong Huang and Jie Wu, "A Probabilistic Clustering Algorithm in Wireless Sensor Networks", Part by NSF grants CCR 0329741, CNS 0434533, CNS 0422762, and EIA 0130806, IEEE Vehicular Technology Conference, 2005.

[5] OssamaYounis and Sonia Fahmy, "Distributed Clustering in Ad-hoc Sensor Networks: A Hybrid, Energy-Efficient Approach", Purdue University, Proceedings of the 6th ACM international symposium, 2005.

[6] Jason H. Li, Miao Yu, Renato Levy, Anna Teittinen, “A Mobility-Resistant Efficient Clustering Approach for ad hoc and sensor networks", ACM SIGMOBILE Mobile Computing and Communications Review 10.2: 1-12, 2006.

[7] Taran Deep Singh Pawa, "Analysis of Low Energy Adaptive Clustering Hierarchy (LEACH) protocol", National Institute of Technology, Rourkela, 2011.

[8] Chao SHA, Ru-chuan WANG, Hai-ping HUANG, Li juan SUN, "Energy efficient clustering algorithm for data aggregation in wireless sensor networks", Nanjing 
University of Posts and Telecommunications, Nanjing 210003, China, The Journal of China Universities of Posts and Telecommunications, January 2011.

[9] Li Qing, Qingxin Zhu, Mingwen Wang, "Design of a distributed energy-efficient clustering algorithm for heterogeneous wireless sensor networks", Journal of Computer Communications 29.12: 2230-2237, 2006.

[10] Shio Kumar Singh, M P Singh, D K Singh, "Routing Protocols in Wireless Sensor Networks- A Survey", International Journal of Computer Science \& Engineering Survey (IJCSES) Vol.1, No.2, November 2010 .

[11] Vinay Kumar Singh and Vidushi Sharma, "Lifetime Maximization of Wireless Sensor Networks using improved Genetic Algorithm Based Approach", Vol-57-

Deutschland, Proceedings of IEEE 66th Vehicular Technology Conference (VTC Fall), October 2008.

[16] RabieA. Ramadan, "Agent Based Multipath Routing in Wireless Sensor Networks", German University in Cairo, Egypt, IEEE Symposium on, pp. 63-69.IEEE, 2009.

[17] Tiago Camilo, Carlos Carreto, Jorge Sá Silva, Fernando Boavida," "An Energy-Efficient Ant-Based Routing Algorithm for Wireless Sensor Networks", Laboratory of Communications and Telematics, University of Coimbra and InstitutoPolitécnico da Guarda, Portugal, 2006.

[18] Luis Cobo, Alejandro Quintero, Samuel Pierre, "Antbased routing for wireless multimedia sensor networks using multiple QoS metrics", Computer networks 54.17, 2991-3010, May 2010.

[19] Alexandre Massayuki Okazaki and Ant`onio Augusto Fr"ohlich, "Ant-based Dynamic Hop Optimization Protocol: a Routing Algorithm for Mobile Wireless Sensor Networks", Laboratory for Software and Hardware Integration (LISHA) Federal University of Santa Catarina (UFSC), i2ts, pp 30-35, 2009.
No.14, International Journal of Computer Applications $57.14,2012$.

[12] Liu Yueyang, Ji Hong, YueGuangxin, "An EnergyEfficient PEGASIS-Based Enhanced Algorithm in Wireless Sensor Networks", DCN Lab, Beijing University of Posts and Telecommunications, Beijing, China Communications: 91-97, 2006.

[13] Juhana Yrjölä, "Energy-Efficient Communication Protocol for Wireless Microsensor Networks", T-79.194 Seminar on theoretical computer science, 2005.

[14] Yan Yu, Ramesh Govindan, Deborah Estrin, "Geographical and Energy Aware Routing: a recursive data dissemination protocol for wireless sensor networks", Technical report ucla/csd-tr-01-0023, UCLA, 2001.

[15] Charles Harsch, Andreas Festag, PanosPapadimitratos, "Secure Position-Based Routing for VANETs",NEC

[20] Wei-Ming Chen, Chung-Sheng Li, Fu-Yu Chiang, HanChieh Chao, "Jumping ant routing algorithm for sensor networks", Journal of Computer Communications, Volume 30, Issue 14-15, 2007.

[21] Dr. Rituparna Chaki, Ayan Kumar Das, Ms. Samarpita Mukherjee, Ms. Rakhi Dutta, "Review Work for Sensor Network", National Conference, at KJ Somaiya College of Engineering, 2009.

[22] Aleksi Ahtiainen, "Summary of Rumor Routing in Wireless Sensor Network", Helsinki University of Technology, Seminar on Theoretical Computer Science, T-79.194, Feb 2005.

[23] Maurice Chu, Horst Haussecker, and Feng Zhao, "Scalable Information-Driven Sensor Querying and Routing for ad hoc Heterogeneous Sensor Networks", Xerox Palo Alto Research Center Technical Report, International Journal of High Performance Computing Applications 16.3: 293-313, 2002 